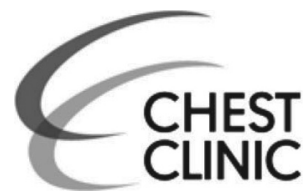

Department of Cardiothoracic Surgery, St Bartholomew's Hospital, London, UK

\section{Correspondence to}

Wael I Awad, Department of Cardiothoracic Surgery, St

Bartholomew's Hospital, West Smithfield, London EC1A 7BE, UK;

wael.awad@bartshealth.nhs.uk

Received 20 September 2016 Revised 5 January 2017

Accepted 6 January 2017 Published Online First

1 February 2017

\section{SLlinked}

- http://dx.doi.org/10.1136/ thoraxjnl-2016-209456

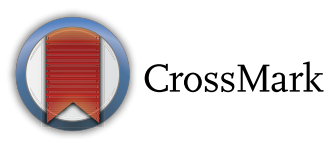

To cite: Nguyen A, Habib $H$, Awad WI. Thorax

2017;72:1060-1061.

IMAGES IN THORAX

\title{
Giant thymic cyst mimicking a unilateral pleural effusion
}

\author{
Anita Nguyen, Hameed Habib, Wael I Awad
}

A 24-year-old woman presented to hospital with a 3 -day history of right-sided chest pain and worsening shortness of breath. On examination, stony dullness of the right side of the chest was noted. Blood tests showed raised inflammatory markers (erythrocyte sedimentation rate $87 \mathrm{~mm} /$ hour, C-reactive protein $338 \mathrm{mg} / \mathrm{L}$ ), but the remainder of the investigations were unremarkable. A chest radiograph was reported as showing a large right-sided pleural effusion with atelectasis of the right lower lobe (figure 1A), with a recommendation for percutaneous drainage by the radiologists. A percutaneous pleural pigtail catheter was inserted, but only $200 \mathrm{~mL}$ of fluid was drained; this showed reactive mesothelial and inflammatory cells, but no evidence of malignancy and negative cultures. A CT scan of the chest subsequently revealed a $21 \times 16 \mathrm{~cm}$ cyst almost filling the right hemithorax (figure 1B-D). The patient was discussed at the multi-disciplinary team meeting where surgical excision of the lesion was recommended.

The patient underwent a right postero-lateral thoracotomy. At surgery, a large tense cystic mass was seen firmly adherent to the lung and pericardium. 1.3 L of serous fluid were drained from the mass to facilitate dissection. The mass extended into the neck and was continuous with the thymus gland. It was then carefully dissected off the lung and pericardium together with both thymic lobes (figure 2A). Care was taken to prevent damage to the phrenic nerve, which was stretched over the surface of the mass but easily separated.

Histopathological assessment showed a large benign cyst with multiple locules. Cuboidal and
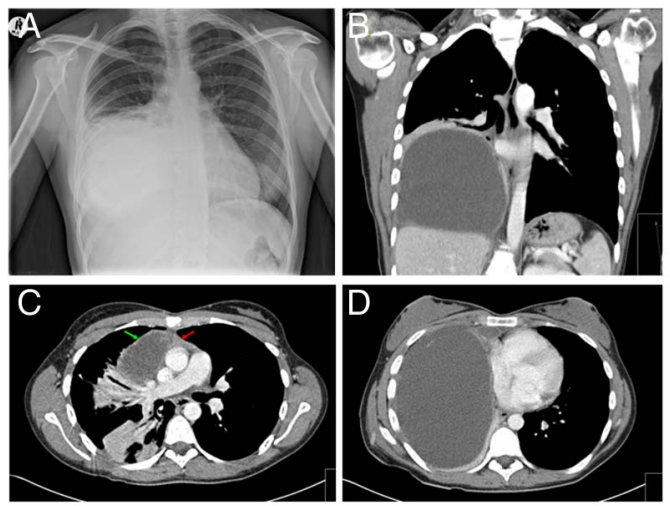

Figure 1 (A) Preoperative chest radiograph showing a large right-sided pleural effusion with compressive atelectasis of the right lower lobe. (B) Coronal view of CT scan showing a $21 \times 16 \mathrm{~cm}$ cyst occupying the right hemithorax. (C) Axial view of CT scan showing the cyst (green arrow) and thymus gland (red arrow). (D) Axial view of CT scan showing the cyst.
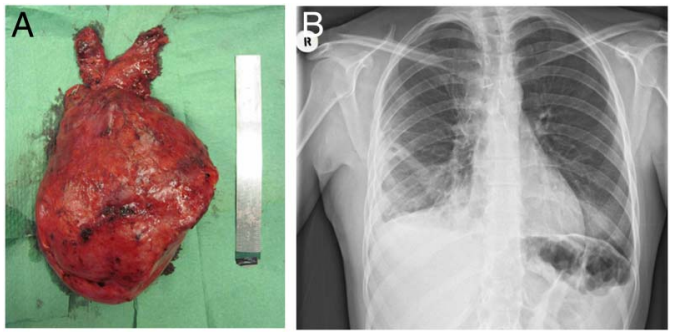

Figure 2 (A) Thymic cyst and thymus resected at surgery following drainage of $1.3 \mathrm{~L}$ of cystic fluid (next to a $15 \mathrm{~cm}$ ruler). (B) Postoperative chest radiograph showing a fully expanded right lung and normal right hemidiaphragm.

pseudostratified epithelial lining of the cyst and mild surrounding chronic inflammation confirmed this to be a multilocular thymic cyst. There was no evidence of neoplasia.

Postoperative chest radiographs showed a fully expanded right lung with a normal right hemidiaphragm (figure 2B). The patient made an uneventful recovery and was discharged from hospital 5 days after surgery. She remains well with no recurrence 5 years postoperatively.

\section{DISCUSSION}

Thymic cysts are a rare cause of mediastinal masses and can be located anywhere between the angle of the jaw and the diaphragm. They are usually asymptomatic, but very large cysts may present with cough, dyspnoea, chest pain or oesophageal compression. ${ }^{12}$

Thymic cysts are either congenital or acquired. Congenital cysts are unilocular and characterised by a thin, translucent wall. Acquired cysts are multilocular thymic cysts (MTCs) with thick walls usually associated with inflammation. ${ }^{3}$

MTCs are thought to be triggered by an inflammatory process of the thymic parenchyma. They have been found in patients with auto-immune diseases (eg, systemic lupus erythematosus, rheumatoid arthritis, Hashimoto's thyroiditis) suggesting immune-mediated inflammation as a likely explanation of the pathophysiological changes in MTC. ${ }^{13}$

The treatment of MTCs remains controversial, and small MTCs can be managed either conservatively or surgically, mainly to confirm diagnosis. However, the consensus on treatment for large MTCs causing compression of surrounding structures is to excise the lesion. ${ }^{2}$ Removal of MTCs may be considered via video-assisted thoracoscopic surgery, open thoracotomy or median sternotomy. 
To our knowledge, no recurrences of MTCs have yet been reported in the literature; however, it is still important to ensure complete resection of an MTC at surgery.

\section{CONCLUSION}

Thymic cysts are usually found incidentally and rarely become large enough to present with compressive symptoms. Our case highlights that thymic cysts can reach massive proportions before presenting acutely, that reported 'pleural effusions' on chest radiography are not always what they appear to be, and an ultrasound examination should be considered prior to a drainage procedure.
Contributors $\mathrm{AN}, \mathrm{HH}$ and WIA wrote the manuscript. WIA supervised the study. All authors approved the final version of the manuscript.

Competing interests None declared.

Provenance and peer review Not commissioned; externally peer reviewed.

\section{REFERENCES}

1 Suster S, Rosai J. Multilocular thymic cyst: an acquired reactive process. Am J Surg Pathol 1991;15:388-98.

2 Jennings S, Stuklis RG, Chan J, et al. Successful giant cyst removal: case report and review of the literature. Heart Lung Circ 2015;24:e89-92.

3 Choi YW, McAdams HP, Jeon SC, et al. Idiopathic multilocular thymic cyst: CT features with clinical and histopathologic correlation. AJR Am J Roentgenol 2001;177:811-15. 